\title{
Special Ruled Surface in de-Sitter 3-Space
}

\author{
Tuğba Mert ${ }^{1 *}$ and Mehmet Atçeken ${ }^{2}$ \\ ${ }^{1}$ Department of Mathematics, Faculty of Science, Cumhuriyet University, Sivas, Turkey \\ ${ }^{2}$ Department of Mathematics, Faculty of Science and Arts, Aksaray University, Aksaray, Turkey \\ ${ }^{*}$ Corresponding author
}

\author{
Article Info \\ Keywords: Base curve, Central curve, \\ Central point, Straight geodesic \\ 2010 AMS: 53A35 \\ Received: 6 May 2021 \\ Accepted: 14 September 2021 \\ Available online: 15 September 2021
}

\begin{abstract}
In this paper, timelike base curve and spacelike main geodesic with the timelike ruled surface are studied, which is a special class of ruled surface in de-Sitter space $S_{1}^{3}$. A ruled surface in the de-Sitter space $S_{1}^{3}$ is obtained by moving a geodesic along a curve. So we will call these surfaces in the de-Sitter space as the geodesic ruled surface. Developable ruled surface, striction point, striction curve, dispersion parameter, and orthogonal trajectory concepts are investigated for the obtained geodesic ruled surface.
\end{abstract}

\section{Introduction}

The de-Sitter space is a model for physical events, and many physical phenomena can be explained by these models. Therefore, the surface varieties in de-Sitter space are very important. The surface types in different spaces guide the areas related to our daily life such as architecture and geometric design and therefore, the ruled surfaces in de-Sitter space are of great importance. It can be seen during history via the Euclidean motif in BC first, then spherical motif in the medieval and hyperbolic motif in the modern times in the architectures. In the future, architectural structures and geometric designs using de-Sitter lines will enter our daily lives. There is more than one causal character for surfaces, curves, and lines of de-Sitter space due to the structure of de-Sitter space. Since the surface of de-Sitter space can be considered as spacelike and timelike, then also curves and lines of de-Sitter space can be considered as spacelike and timelike.

Let $U \subset \mathbb{R}^{2}$ be an open subset, and let $x: U \rightarrow S_{1}^{3}$ be an embedding. If the vector subspace $\tilde{U}$ which generated by $\left\{x_{u_{1}}, x_{u_{2}}\right\}$ contains at least a timelike vector field then $x$ is called timelike surface in $S_{1}^{3}$,i.e., the normal on the surface is a spacelike vector. In [1], Turgut and Hacisalihoglu studied timelike ruled surfaces in the Minkowski-3 space. They showed that these surfaces are obtained by a timelike straight line moving along a spacelike curve. A ruled surface is a surface generated by a straight line $l$ moving along a curve $\alpha$ [1]. The various positions of the generating line $l$ are called the rulings of the surface. Similarly, they studied spacelike ruled surfaces in the Minkowski-3 space [2]. Sabuncuoğlu studied generalized ruled surfaces in Euclidean $n$-space $E^{n}$ and showed that the necessary and sufficient condition for the $n$-dimensional ruled surface to be a minimal surface is that the curves perpendicular to the rectangular space are asymptotic curves [3]. Later, Mert introduced spacelike ruled surfaces in the hyperboloid model of hyperbolic 3-space in Minkowski space, and using the properties of hyperbolic space, she investigated the properties of these type ruled surfaces [4].

Let $x: M \longrightarrow \mathbb{R}_{1}^{4}$ be an immersion of a surface $M$ into $\mathbb{R}_{1}^{4}$. We say that $x$ is timelike (resp. spacelike, lightlike) if the induced metric on $M$ via $x$ is Lorentzian (resp. Riemannian, degenerated). If $\langle x, x\rangle=1$, then $x$ is an immersion of $S_{1}^{3}$ [5]. Since geodesic which is lines of de-Sitter space on a ruled surface can be obtained by moving of curves in space, a sort of ruled surface can be captured up to causal characters of the base curve and main geodesic. In this paper, we investigate timelike ruled surfaces which have a base curve as timelike and main geodesic as spacelike in de-Sitter space $S_{1}^{3}$. A ruled surface is a surface obtained by a geodesic $d_{s}^{\alpha}$ moving along a curve $\alpha$. Therefore, such surfaces may also be called geodesic ruled 
surfaces. Thus, the geodesic ruled surface has a parameterization in $S_{1}^{3}$ as follows

$$
\varphi(s, t)=(\cos t) \alpha(s)+(\sin t) Z(s)
$$

where $\alpha$ is called the base curve and $Z$ is called the director vector of $d_{s}^{\alpha}$. If the tangent plane is constant along with a fixed ruling, then the ruled surface is called a developable geodesic ruled surface.

\section{Preliminaries}

For basic notions and properties of the Lorentz-Minkowski space from the viewpoint of Lorentz geometry, see [6]. Let $R_{1}^{4}$ be 4-dimensional vector space equipped with the scalar product $\langle$,$\rangle which is defined by$

$$
\langle x, y\rangle=-x_{1} y_{1}+x_{2} y_{2}+x_{3} y_{3}+x_{4} y_{4} .
$$

Then, $R_{1}^{4}$ is called Lorentzian 4- space or 4-dimensional Minkowski space. The Lorentzian norm (length) of $x$ is defined to be

$$
\|x\|=|\langle x, x\rangle|^{\frac{1}{2}} .
$$

If $\left(x_{1}^{i}, x_{2}^{i}, x_{3}^{i}, x_{5}^{i}\right)$ is the coordinate of $x_{i}$ with respect to canonical basis $\left\{e_{1}, e_{2}, e_{3}, e_{4}\right\}$ of $R_{1}^{4}$, then the lorentzian cross product $x_{1} \times x_{2} \times x_{3}$ is defined by the symbolic determinant

$$
x_{1} \times x_{2} \times x_{3}=\left|\begin{array}{cccc}
-e_{1} & e_{2} & e_{3} & e_{4} \\
x_{1}^{1} & x_{2}^{1} & x_{3}^{1} & x_{4}^{1} \\
x_{1}^{2} & x_{2}^{2} & x_{3}^{2} & x_{4}^{2} \\
x_{1}^{3} & x_{2}^{3} & x_{3}^{3} & x_{4}^{3}
\end{array}\right| .
$$

One can easily see that

$$
\left\langle x_{1} \times x_{2} \times x_{3}, x_{4}\right\rangle=\operatorname{det}\left(x_{1}, x_{2}, x_{3}, x_{4}\right) .
$$

Given a vector $v \in R_{1}^{4}$ and a real number $c$, the hyperplane with pseudonormal $v$ is defined by

$$
H P(v, c)=\left\{x \in \mathbb{R}_{1}^{4} \mid\langle x, v\rangle=c\right\}
$$

We say that $\operatorname{HP}(v, c)$ is a spacelike hyperplane, timelike hyperplane or lightlike hyperplane if $v$ is timelike, spacelike or lightlike, respectively. We have the following three types of pseudo-spheres in $R_{1}^{4}$ :

$$
\begin{array}{rc}
\text { Hyperbolic-3 space } & : H^{3}(-1)=\left\{x \in R_{1}^{4} \mid\langle x, x\rangle=-1, x_{0} \geq 1\right\}, \\
\text { de Sitter 3- space } & : S_{1}^{3}=\left\{x \in R_{1}^{4} \mid\langle x, x\rangle=1\right\}, \\
\text { (open) lightcone } & : L C^{*}=\left\{x \in R_{1}^{4} \backslash\{0\} \mid\langle x, x\rangle=0, x_{0}>0\right\} .
\end{array}
$$

We also define the lightcone 3 -sphere

$$
S_{+}^{3}=\left\{x=\left(x_{1}, x_{2}, x_{3}, x_{4}\right) \mid\langle x, x\rangle=0, x_{1}=1\right\} .
$$

A hypersurface given by the intersection of $S_{1}^{3}$ with a spacelike (resp.timelike) hyperplane is called an elliptic hyperquadric (resp. hyperbolic hyperquadric). If $c \neq 0$ and $H P(v, c)$ are lightlike, then $H P(v, c) \cap S_{1}^{3}$ is a de Sitter horosphere.

In the point of view of Kasedou [7], we construct the extrinsic differential geometry of curves in $S_{1}^{3}$. Since $S_{1}^{3}$ is a Riemannian manifold, the regular curve $\gamma: I \rightarrow S_{1}^{3}$ is given by the arclength parameter.

Theorem 2.1. i) If $\gamma: I \rightarrow S_{1}^{3}$ is a spacelike curve with unit speed, then Frenet-Serret type formulae are obtained

$$
\left\{\begin{array}{l}
\gamma^{\prime}(s)=t(s) \\
t^{\prime}(s)=\kappa_{d}(s) n(s)-\gamma(s) \\
n^{\prime}(s)=-\kappa_{d}(s) t(s)-\tau_{d}(s) e(s) \\
e^{\prime}(s)=-\tau_{d}(s) n(s)
\end{array}\right.
$$

where

$$
\kappa_{d}(s)=\left\|t^{\prime}(s)+\gamma(s)\right\|
$$

and

$$
\tau_{d}(s)=-\frac{\operatorname{det}\left(\gamma(s), \gamma^{\prime}(s), \gamma^{\prime \prime}(s), \gamma^{\prime \prime \prime}(s)\right)}{\left(\kappa_{d}(s)\right)^{2}}
$$


in [8].

ii) If $\gamma: I \rightarrow S_{1}^{3}$ is a timelike curve with unit speed, then Frenet-Serret type formulae are obtained

$$
\left\{\begin{array}{l}
\gamma^{\prime}(s)=t(s) \\
t^{\prime}(s)=\kappa_{d}(s) n(s)+\gamma(s) \\
n^{\prime}(s)=-\kappa_{d}(s) t(s)+\tau_{d}(s) e(s) \\
e^{\prime}(s)=-\tau_{d}(s) n(s)
\end{array}\right.
$$

where

$$
\kappa_{d}(s)=\left\|t^{\prime}(s)-\gamma(s)\right\|
$$

and

$$
\tau_{d}(s)=-\frac{\operatorname{det}\left(\gamma(s), \gamma^{\prime}(s), \gamma^{\prime \prime}(s), \gamma^{\prime \prime \prime}(s)\right)}{\left(\kappa_{d}(s)\right)^{2}}
$$

in [8].

It is easily seen that $\kappa_{d}(s)=0$ if and only if there exists a lightlike vector $c$ such that $\gamma(s)-c$ is a geodesic.

Now we give extrinsic differential geometry on surfaces in $S_{1}^{3}$ due to Kasedou [7].

Let $U \subset \mathbb{R}^{2}$ is an open subset, and $x: U \rightarrow S_{1}^{3}$ is a regular surface $M=x(U)$. Since $M$ is a timelike surface, there is

$$
e(u)=\frac{x(u) \wedge x_{u_{1}}(u) \wedge x_{u_{2}}(u)}{\left\|x(u) \wedge x_{u_{1}}(u) \wedge x_{u_{2}}(u)\right\|}
$$

such that

$$
\langle e, x\rangle \equiv\left\langle e, x_{u_{i}}\right\rangle \equiv 0,\langle e, e\rangle=1 .
$$

Thus there is de Sitter Gauss image of $x$ which is defined by mapping $E: U \rightarrow S_{1}^{3}$,

$$
E(u)=e(u) .
$$

The lightcone Gauss image of $x$ is defined by map $L^{ \pm}: U \rightarrow L C^{*}$,

$$
L^{ \pm}(u)=x(u) \pm e(u) .
$$

The derivative $d x\left(u_{0}\right)$ can be identified by the mapping $1_{T_{p} M}$ on the tangent space $T_{p} M$. Therefore, we have

$$
d L^{ \pm}\left(u_{0}\right)=1_{T_{p} M} \pm d E\left(u_{0}\right) .
$$

The linear transformations

$$
S_{p}^{ \pm}:=-d L^{ \pm}\left(u_{0}\right): T_{p} M \rightarrow T_{p} M
$$

and

$$
A_{p}:=-d E\left(u_{0}\right): T_{p} M \rightarrow T_{p} M
$$

are called the hyperbolic shape operator and de Sitter shape operator of $M$ at $p=x\left(u_{o}\right)$, respectively.

Let $\bar{K}_{i}^{ \pm}(p)$ and $K_{i}(p),(i=1,2)$ be the eigenvalues of $S_{p}^{ \pm}$and $A_{p}$. Since

$$
S_{p}^{ \pm}=-1_{T_{p} M} \pm A_{p},
$$

$S_{p}^{ \pm}$and $A_{p}$ have the same eigenvectors and relations

$$
\bar{K}_{i}^{ \pm}(p)=-1 \pm K_{i}(p) .
$$

$\bar{K}_{i}^{ \pm}(p)$ and $K_{i}(p),(i=1,2)$ are called hyperbolic and de Sitter principal curvatures of $M$ at $p$, respectively.

Let $\gamma(s)$ be a unit speed curve on $M$, with $p=\gamma\left(u_{1}\left(s_{0}\right), u_{2}\left(s_{0}\right)\right)$. We consider the hyperbolic curvature vector

$$
k(s)=t^{\prime}(s)-\gamma(s)
$$


and the de Sitter normal curvature

$$
K_{n}^{ \pm}\left(s_{0}\right)=\left\langle k\left(s_{0}\right), L^{ \pm}\left(u_{1}\left(s_{0}\right), u_{2}\left(s_{0}\right)\right)\right\rangle=\left\langle t^{\prime}\left(s_{0}\right), L^{ \pm}\left(u_{1}\left(s_{0}\right), u_{2}\left(s_{0}\right)\right)\right\rangle+1
$$

of $p=\gamma\left(u_{1}\left(s_{0}\right), u_{2}\left(s_{0}\right)\right)$. The de Sitter normal curvature depends only on the point $p$ and the unit tangent vector of $M$ at $p$. The hyperbolic normal curvature of $\gamma(s)$ is defined to be

$$
\bar{K}_{n}^{ \pm}(s)=K_{n}^{ \pm}(s)-1 .
$$

The extrinsic (de Sitter) Gauss curvature and mean curvature of $M$ at $p$ is given by

$$
K_{e}\left(u_{0}\right)=\operatorname{det} A_{p}=K_{1}(p) K_{2}(p)
$$

and

$$
K_{d}\left(u_{0}\right)=\frac{1}{2} \operatorname{TraceA}_{p}=\frac{K_{1}(p)+K_{2}(p)}{2} .
$$

\section{3. $T S$-geodesic ruled surface in de-Sitter 3-space}

Now let's investigate the timelike ruled surfaces that its base curve is a timelike curve and its direction geodesic is a spacelike geodesic in the de-Sitter space $S_{1}^{3}$. Hereinafter, in terms of brevity, we call the $T S$-geodesic ruled surfaces the geodesic ruled surfaces whose base curve is timelike and the direction geodesic is spacelike.

Let $\alpha$ be a differentiable timelike curve with the unit speed in de-Sitter space $S_{1}^{3}$, then it is defined by

$$
\alpha: I \rightarrow S_{1}^{3} \subset R_{1}^{4}, \alpha(s)=\left(\alpha_{1}(s), \alpha_{2}(s), \alpha_{3}(s), \alpha_{4}(s)\right), \forall s \in I
$$

where $\{0\} \subset I \subset \mathbb{R}$. In here

$$
\langle\alpha(s), \alpha(s)\rangle=1
$$

and since $\alpha$ base curve is a timelike curve, we have

$$
\left\langle\alpha^{\prime}(s), \alpha^{\prime}(s)\right\rangle=-1 .
$$

Let's assume that

$$
\langle\alpha(s), Z(s)\rangle=0, \forall s \in I
$$

where

$$
Z: I \rightarrow S_{1}^{3}, Z(s)=\left(z_{1}(s), z_{2}(s), z_{3}(s), z_{4}(s)\right)
$$

and

$$
\langle Z(s), Z(s)\rangle=1 .
$$

Then, a geodesic $d_{s}^{\alpha}$ in de-Sitter space $S_{1}^{3}$ has a parametrization

$$
d_{s}^{\alpha}: \mathbb{R} \rightarrow S_{1}^{3}, d_{s}^{\alpha}(t)=(\cos t) \alpha(s)+(\sin t) Z(s)
$$

where $\alpha(s)$ is a initial point and $Z(s)$ is the direction vector of $d_{s}^{\alpha}$ [6]. Here frenet components of base curve $\alpha(s)$ are $\left\{T_{\alpha}, N_{\alpha}, B_{\alpha}, \kappa_{d}, \tau_{d}\right\}$. Let $T_{d}$ be tangent of geodesic $d_{s}^{\alpha}$ at the point $\alpha(s)$ and assume that $T_{d}$ and $T_{\alpha}$ are linearly independent for all $s \in I$. Then, we obtain $(I \times \mathbb{R}, \varphi)$ parametrized by $\varphi: I \times \mathbb{R} \rightarrow S_{1}^{3}$

$$
\varphi(s, t)=(\cos t) \alpha(s)+(\sin t) Z(s) .
$$

This $(I \times \mathbb{R}, \varphi)$ surface is called a geodesic ruled surface which is produced by the geodesic $d_{s}^{\alpha}$. Let us denote this geodesic ruled surface with $M$. Then we can give the following definition.

Definition 3.1. The surface obtained by moving a given $d_{s}^{\alpha}$ spacelike geodesic along a given $\alpha$ timelike curve is called the $T S$-geodesic ruled surface in the de-Sitter space $S_{1}^{3}$, where $d_{s}^{\alpha}$ is the direction geodesic of the TS-geodesic ruled surface and the $\alpha$ curve is called the base curve of TS-geodesic ruled surface. 
Let us find the orthonormal base of tangent space $\chi(M)$ of geodesic ruled surface $M$ along the timelike curve $\alpha$. If $T$ is a unit tangent vector of timelike curve $\alpha$ and $Z$ is the unit director vector of spacelike geodesic $d_{s}^{\alpha}$, then we can choose spacelike vector field such that

$$
Y=\tilde{T}_{d}+\left\langle\tilde{T}_{d}, T\right\rangle T
$$

that is orthogonal to $T$ in this plane, where

$$
\tilde{T}_{d}=\frac{T_{d}}{\left\|T_{d}\right\|}
$$

is the unit tangent of geodesic $d_{s}^{\alpha}$ and

$$
T_{d}=(\cos t) T_{\alpha(s)}+(\sin t) T_{Z(s)}
$$

Also, if we take

$$
X=\frac{Y}{\|Y\|}
$$

then

$$
\|X\|=1,\langle X, T\rangle=0 \text { and }\langle T, T\rangle=-1 .
$$

Thus, $\{X, T\}$ are the orthonormal vectors of $\chi(M)$. Also,

$$
\xi=\varphi \times T \times X
$$

is the normal vector of $T S$-geodesic ruled surface $M$ in de-Sitter space $S_{1}^{3}$, that is

$$
\begin{array}{rc}
\xi & \in \chi^{\perp}(M) \\
\chi\left(S_{1}^{3}\right) & =S p\{X, T\} \oplus S p\{\xi\} \\
\chi\left(R_{1}^{4}\right) & =S p\{X, T\} \oplus \operatorname{Sp}\{\xi, \varphi\}
\end{array}
$$

In this case, system $\{\varphi, T, X, \xi\}$ is the orthonormal base of $M$.

Now let investigate the alteration of this system along the timelike curve $\alpha$. The Levi-Civita connection of $R_{1}^{4}, S_{1}^{3}$, and $M$ is denoted $\overline{\bar{D}}, \bar{D}$, and $D$, respectively. Then we have the Gauss formulas [9]

$$
\begin{cases}\overline{\bar{D}}_{X} Y=\bar{D}_{X} Y-\langle X, Y\rangle \alpha & , \tilde{A}(X)=\overline{\bar{D}}_{X} \alpha=I(X) \\ \bar{D}_{X} Y=D_{X} Y-\langle A(X), Y\rangle \xi & , A(X)=\bar{D}_{X} \xi\end{cases}
$$

In de-Sitter space $S_{1}^{3}$, let's derive the $\{T, X, \xi\}$ orthonormal frame along timelike curve $\alpha$. In this case, we get the system in $S_{1}^{3}$

$$
\begin{cases}\bar{D}_{T} T & =a X+b \xi \\ \bar{D}_{T} X & =a T+c \xi \\ \bar{D}_{T} \xi & =b T-c X\end{cases}
$$

The matrix representation of this system is

$$
\left[\begin{array}{c}
\bar{D}_{T} T \\
\bar{D}_{T} X \\
\bar{D}_{T} \xi
\end{array}\right]=\left[\begin{array}{ccc}
0 & a & b \\
a & 0 & c \\
b & -c & 0
\end{array}\right]\left[\begin{array}{c}
T \\
X \\
\xi
\end{array}\right]
$$

where

$$
a=\left\langle\bar{D}_{T} T, X\right\rangle, b=\left\langle\bar{D}_{T} T, \xi\right\rangle \text { and } c=\left\langle\bar{D}_{T} X, \xi\right\rangle
$$

Now, in $R_{1}^{4}$, let's derive the $\{\varphi, T, X, \xi\}$ orthonormal frame along timelike curve $\alpha$. In this case, we get the system

$$
\begin{cases}\overline{\bar{D}}_{T} \varphi & =(\cos t+a \sin t) T+(c \sin t) \xi \\ \overline{\bar{D}}_{T} T & =\varphi+a X+c \xi \\ \overline{\bar{D}}_{T} X & =a T+c \xi \\ \bar{D}_{T} \xi & =b T-c X\end{cases}
$$


in $R_{1}^{4}$. System 3.1 have the for matrix form

$$
\left[\begin{array}{c}
\overline{\bar{D}}_{T} \varphi \\
\overline{\bar{D}}_{T} T \\
\overline{\bar{D}}_{T} X \\
\overline{\bar{D}}_{T} \xi
\end{array}\right]=\left[\begin{array}{cccc}
0 & \cos t+a \sin t & 0 & c \sin t \\
1 & 0 & a & b \\
0 & a & 0 & c \\
0 & b & -c & 0
\end{array}\right]\left[\begin{array}{c}
\varphi \\
T \\
X \\
\xi
\end{array}\right]
$$

For ruled surface $M$ that is given by parametrization

$$
\begin{gathered}
\varphi: I \times \mathbb{R} \rightarrow S_{1}^{3}, \varphi(s, t)=(\cos t) \alpha(s)+(\sin t) X(s) \\
\left\{\begin{aligned}
E & =\left\langle\varphi_{s}, \varphi_{s}\right\rangle=-(\cos t+a \sin t)^{2}+c^{2} \sin ^{2} t \\
F & =\left\langle\varphi_{s}, \varphi_{t}\right\rangle=0 \\
G & =\left\langle\varphi_{t}, \varphi_{t}\right\rangle=1,
\end{aligned}\right.
\end{gathered}
$$

where

$$
\langle\xi, \xi\rangle=F^{2}-E G=-E .
$$

Since $\xi$ is the spacelike vector that is

$$
\langle\xi, \xi\rangle>0,
$$

then the geodesic ruled surface is a timelike surface and

$$
E<0 .
$$

Let us the denote domain of $t$ by

$$
\begin{gathered}
J=\{t \mid E=E(t)<0\} . \\
\varphi_{t_{o}}: I \times\left\{t_{0}\right\} \rightarrow M, \varphi_{t_{0}}\left(s, t_{0}\right)=\left(\cos t_{0}\right) \alpha(s)+\left(\sin t_{0}\right) X(s)
\end{gathered}
$$

determines a curve of $T S$-geodesic ruled surface $M$ where $t$ is constant in its domain. The tangent vector field of this curve is

$$
A=\left(\cos t_{0}+a \sin t_{0}\right) T(s)+c\left(\sin t_{0}\right) \xi(s) .
$$

Since

$$
\langle A, A\rangle=E
$$

and

$$
E<0,
$$

then $A$ is a timelike vector. Thus $\varphi_{t_{0}}$ curve is a timelike curve and also

$$
\langle X, A\rangle=0
$$

Remark 3.2. Since the stereographic projection is a conformal map, using stereographic projection, the following example can be provided from [10].

Example 3.3. Let us take $T S-$ geodesic ruled surface $M$ in de-Sitter space $S_{1}^{3}$ given by parametrization

$$
\varphi: I \times \mathbb{R} \rightarrow S_{1}^{3}, \varphi(s, t)=(\cos t) \alpha(s)+(\sin t) X(s) .
$$

In here, if

$$
\alpha(s)=(\sinh s, 0, \cosh s, 0)
$$

and

$$
X(s)=(-\cosh s, \sqrt{2}, \sinh s, 0)
$$

are chosen, then $\varphi(s, t)$ is TS-geodesic ruled surface in de-Sitter space $S_{1}^{3}$. 


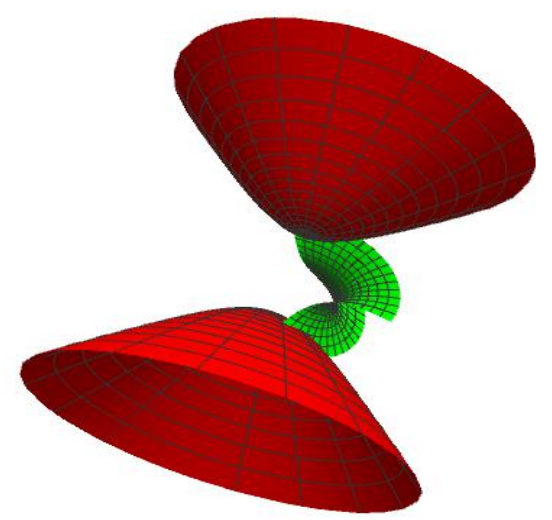

Figure 3.1: Timelike Geodesic Ruled Surface in de-Sitter 3-Space

\section{Developable timelike geodesic ruled surfaces}

Definition 4.1. If the tangent planes of a TS-geodesic ruled surface in $S_{1}^{3}$ are the same along its main geodesics, then this timelike ruled surface is called a developable timelike geodesic ruled surface.

Theorem 4.2. Let $M$ be timelike ruled surface whose are base curve as timelike and main geodesic as spacelike in de-Sitter space $S_{1}^{3}$. Then the tangent planes are the same along the main geodesic if and only if $c=0$.

Proof. Let $M$ be a $T S$-geodesic ruled surface in de-Sitter space $S_{1}^{3}$, and suppose that tangent planes of this ruled surface are the same along with one of its main geodesics. We consider the tangent vector field

$$
A=\left(\cos t_{0}+a \sin t_{0}\right) T(s)+c\left(\sin t_{0}\right) \xi(s)
$$

of curve $\varphi_{t_{0}}: I \times\left\{t_{0}\right\} \rightarrow M$ which is at $t_{0} \in I$. Since $\varphi_{t_{0}}$ is the parameter curve of $M$, the vector $A$ is in the tangent plane of the surface $M$. Hence

$$
c=0
$$

Conversely, assume that

$$
c=0 \text {. }
$$

In this case, since

$$
A=\left(\cos t_{0}+a \sin t_{0}\right) T(s)
$$

and

$$
T_{\varphi\left(t_{0}, s\right)} M=\operatorname{sp}\{T, X\}=\operatorname{sp}\{T, A\} .
$$

This means that the tangent planes are the same along with one of its main geodesics.

Corollary 4.3. The TS-geodesic ruled surface $M$ in de-Sitter space $S_{1}^{3}$ is a developable surface if and only if $c=0$.

Corollary 4.4. For $T S$-geodesic ruled surface $M$ in de-Sitter space $S_{1}^{3}$,

$$
b=-\operatorname{det}\left(T, X, \varphi, \overline{\bar{D}}_{T} T\right) \text { and } c=-\operatorname{det}\left(T, X, \varphi, \overline{\bar{D}}_{T} X\right)
$$

Example 4.5. The surface of example-1 above is an example of a developable ruled surface in de-Sitter space $S_{1}^{3}$. Really, for timelike geodesic ruled surface $M$ in de-Sitter space $S_{1}^{3}$ given by parametrization

$$
\varphi: I \times \mathbb{R} \rightarrow S_{1}^{3}, \varphi(s, t)=(\cos t) \alpha(s)+(\sin t) X(s)
$$

if

$$
\alpha(s)=(\sinh s, 0, \cosh s, 0)
$$


and

$$
X(s)=(-\cosh s, \sqrt{2}, \sinh s, 0)
$$

are chosen, then

$$
c=-\operatorname{det}\left(T, X, \varphi, \overline{\bar{D}}_{T} X\right)=\left|\begin{array}{cccc}
\cosh s & -\sin \left(\frac{s}{\sqrt{2}}\right) & \cos \left(\frac{s}{\sqrt{2}}\right) & 0 \\
-\cosh s & -\sin s & \cos s & 0 \\
\cos t \sinh s-\sin t \cosh s & \sqrt{2} \sin t & \cos t \cosh s+\sin t \sinh s & 0 \\
-\sinh s & 0 & \cosh s & 0
\end{array}\right| .
$$

Therefore, it is clear that

$$
c=0 .
$$

\section{A striction point and position vector of a striction point}

Definition 5.1. Let TS-geodesic ruled surface be given in de-Sitter space $S_{1}^{3}$. If there exists a common perpendicular of two neighbors the main geodesic of timelike geodesic ruled surface the foot of this perpendicular on principal geodesic is called striction point.

Definition 5.2. When the main geodesic of TS-geodesic ruled surface in de-Sitter space $S_{1}^{3}$ creates the timelike geodesic ruled surface through the base curve, the geometrical place of the striction points of the ruled surface is called the striction curve of $M$.

If $w$ be the distance between the striction point of the timelike geodesic ruled surface and base curve, then position vector $\bar{\alpha}(s)$ can be defined by

$$
\bar{\alpha}(s, w)=(\cos w) \alpha(s)+(\sin w) X(s)
$$

where $\alpha(s)$ is the position vector of the timelike base curve and $X(s)$ is the direction vector of the spacelike main geodesic. The parameter $w$ can be written as the combination of the position vector of the base curve and direction vector of the timelike geodesic ruled surface. Let the first two of three neighbor geodesic of the timelike ruled surface be

$$
d_{s}^{\alpha}=(\cos t) \alpha(s)+(\sin t) X(s)
$$

and

$$
d_{s+\Delta s}^{\alpha}=(\cos t) \alpha(s+\Delta s)+(\sin t) X(s+\Delta s)
$$

where $X(s)$ and $X(s)+\bar{D}_{T(s)} X(s)$ are the direction vectors of these main geodesic, respectively. Also let $P, P^{\prime}$ and $Q, Q^{\prime}$ be the feet on the main geodesic of the common perpendicular of the neighbor geodesic. Thus $P$ and $Q$ are two different striction points. The direction of common perpendicular first two main geodesics are linearly dependent to the vector

$$
\alpha(s) \times X(s) \times\left[X(s)+\bar{D}_{T(s)} X(s)\right] .
$$

Therefore

$$
\alpha(s) \times X(s) \times\left[X(s)+\bar{D}_{T(s)} X(s)\right]=\alpha(s) \times X(s) \times \bar{D}_{T(s)} X(s) .
$$

The vector $\overrightarrow{P Q}$ coincides with the vector $\overrightarrow{P P^{\prime}}$ in the limiting position, and $\overrightarrow{P Q}$ will be the tangent vector of the striction curve. Since

$$
\langle X(s), \overrightarrow{P Q}\rangle=0 \text { and }\left\langle X(s)+\bar{D}_{T(s)} X(s), \overrightarrow{P Q}\right\rangle=0
$$

we obtain

$$
\left\langle\bar{D}_{T(s)} X(s), \overrightarrow{P Q}\right\rangle=0
$$

Thus we get

$$
\left\langle\bar{D}_{T(s)} X(s), \bar{D}_{T(s)} \bar{\alpha}(s)\right\rangle=0 .
$$

On the other hand, since

$$
\bar{D}_{T(s)} \bar{\alpha}(s)=\overline{\bar{D}}_{T(s)} \bar{\alpha}(s)+\langle T(s), \bar{\alpha}(s)\rangle \bar{\alpha}(s)
$$


we obtain

$$
\bar{D}_{T(s)} \bar{\alpha}(s)=\overline{\bar{D}}_{T(s)} \bar{\alpha}(s)
$$

Consequently, from 5.1, we have

$$
\left\langle\overline{\bar{D}}_{T(s)} X(s), \overline{\bar{D}}_{T(s)} \bar{\alpha}(s)\right\rangle=0
$$

and then

$$
\frac{\sin w}{\cos w}=\frac{a}{-a^{2}+c^{2}}
$$

that is

$$
w=\arctan \left(\frac{a}{-a^{2}+c^{2}}\right)
$$

and

$$
\cos w=\frac{-a^{2}+c^{2}}{\sqrt{a^{2}+\left(-a^{2}+c^{2}\right)^{2}}}, \sin w=\frac{a}{\sqrt{a^{2}+\left(-a^{2}+c^{2}\right)^{2}}} .
$$

So, the position vector of the striction curve is

$$
\bar{\alpha}(s)=\left(\frac{-a^{2}+c^{2}}{\sqrt{a^{2}+\left(-a^{2}+c^{2}\right)^{2}}}\right) \alpha(s)+\left(\frac{a}{\sqrt{a^{2}+\left(-a^{2}+c^{2}\right)^{2}}}\right) X(s) .
$$

Theorem 5.3. The distance between the striction point of the timelike geodesic ruled surface and base curve is constant, that is

$$
w=\arctan \left(\frac{a}{-a^{2}+c^{2}}\right)
$$

Proof. Since

$$
\langle X(s), P Q\rangle=0
$$

we obtain

$$
\left\langle X(s), \bar{D}_{T(s)} \bar{\alpha}(s)\right\rangle=0
$$

and

$$
\bar{D}_{T(s)} \bar{\alpha}(s)=\overline{\bar{D}}_{T(s)} \bar{\alpha}(s) .
$$

Thus

$$
\left\langle X(s), \overline{\bar{D}}_{T(s)} \bar{\alpha}(s)\right\rangle=0
$$

and

$$
(\cos w) \frac{d w}{d s}=0
$$

which implies that

$$
\frac{d w}{d s}=0
$$

and so, $w$ is constant.

Theorem 5.4. The striction curve of an undevelopable TS-geodesic ruled surface in de-Sitter space $S_{1}^{3}$ is independent from choosing base curve. 
Proof. Let us denote two $T S$-geodesic ruled surface in de-Sitter space $S_{1}^{3}$ by

$$
\begin{aligned}
& \varphi(t, s)=(\cos t) \alpha(s)+(\sin t) X(s) \\
& \varphi(t, s)=(\cos t) \beta(s)+(\sin t) X(s)
\end{aligned}
$$

where $\alpha$ and $\beta$ are two different base curves of the timelike geodesic ruled surface in $S_{1}^{3}$. Then the striction curves of timelike geodesic ruled surface are

$$
\begin{aligned}
& \bar{\alpha}(s)=\left(\frac{-a^{2}+c^{2}}{\sqrt{a^{2}+\left(-a^{2}+c^{2}\right)^{2}}}\right) \alpha(s)+\left(\frac{a}{-\sqrt{a^{2}+\left(-a^{2}+c^{2}\right)^{2}}}\right)(\cos t) X(s) \\
& \bar{\beta}(s) \quad=\left(\frac{-a^{2}+c^{2}}{\sqrt{a^{2}+\left(-a^{2}+c^{2}\right)^{2}}}\right) \beta(s)+\left(\frac{a}{-\sqrt{a^{2}+\left(-a^{2}+c^{2}\right)^{2}}}\right) X(s)
\end{aligned}
$$

If we subtract $\bar{\beta}(s)$ from $\bar{\alpha}(s)$ and use 5.2, we obtain

$$
\bar{\alpha}(s)-\bar{\beta}(s)=0
$$

which gives up the proof.

Theorem 5.5. Let $M$ be undevelopable TS-geodesic ruled surface in de-Sitter space $S_{1}^{3}$. The point $\varphi\left(s, v_{0}\right)$ is striction point on the main geodesic which passes through $\alpha(s)$ point if and only if $\bar{D}_{T(s)} X(s)$ is a normal vector of the tangent plane on $\varphi\left(s, v_{0}\right)$ point.

Proof. Let $M$ be undevelopable $T S$-geodesic ruled surface in de-Sitter space $S_{1}^{3}$. Suppose that $\bar{D}_{T(s)} X(s)$ is a normal vector of the tangent plane on $\varphi\left(s, v_{0}\right)$ point. Since the tangent vector field of $\varphi_{v_{0}}: I \times\left\{v_{0}\right\} \rightarrow M$ given by

$$
A=\left(\cos v_{0}+a \sin v_{0}\right) T(s)+c\left(\sin v_{0}\right) \xi(s),
$$

then

$$
\left\langle\bar{D}_{T(s)} X(s), A\right\rangle=0
$$

Thus, we obtain

$$
\frac{\sin v_{o}}{\cos v_{0}}=\frac{a}{-a^{2}+c^{2}} .
$$

Therefore $\varphi\left(s, v_{0}\right)$ is a striction point of $M$.

Conversely, suppose that $\varphi\left(s, v_{0}\right)$ is a striction point with main geodesic passing through the point $\alpha(s)$. Thus, we have

$$
\begin{gathered}
\left\langle\bar{D}_{T(s)} X(s), X(s)\right\rangle=0, \\
\left\langle\bar{D}_{T(s)} X(s), A\right\rangle=-a\left(\cos v_{0}+a \sin v_{0}\right)+c^{2} \sin v_{0} .
\end{gathered}
$$

Since $\varphi\left(s, v_{0}\right)$ is striction point, then we get

$$
-a\left(\cos v_{0}+a \sin v_{0}\right)+c^{2} \sin v_{0}=0 .
$$

Hence, we obtain

$$
\left\langle\bar{D}_{T(s)} X(s), A\right\rangle=0
$$

So, $\bar{D}_{T(s)} X(s)$ is a normal vector of tangent plane at $\varphi\left(s, v_{0}\right)$.

Remark 5.6. Let $\bar{D}_{T(s)} X(s)$ be a normal vector of the tangent plane on the striction point. From the equality, we conclude that

$$
\left\langle\bar{D}_{T(s)} X(s), \bar{D}_{T(s)} X(s)\right\rangle=-a^{2}+c^{2},
$$

i) If $-a^{2}+c^{2}>0$, then $\bar{D}_{T(s)} X(s)$ is a spacelike normal vector field.

ii) If $-a^{2}+c^{2}<0$, then $\bar{D}_{T(s)} X(s)$ is a timelike normal vector field. 
Theorem 5.7. Let $M$ be undevelopable TS-geodesic ruled surface in de-Sitter space $S_{1}^{3}$. The striction curve $\bar{\alpha}(s)$ has the form.

i) If $-a^{2}+c^{2}>0$, then the striction curve $\bar{\alpha}(s)$ is a timelike curve.

ii) If $-a^{2}+c^{2}<0$, then the striction curve $\bar{\alpha}(s)$ is a spacelike curve.

Proof. We need to show that the tangent vector field of striction curve $\bar{\alpha}$ is a spacelike vector field or timelike vector field. It is clear that

$$
\left\langle\overline{\bar{D}}_{T(s)} \bar{\alpha}(s), \overline{\bar{D}}_{T(s)} \bar{\alpha}(s)\right\rangle=\frac{-c^{2}}{-a^{2}+c^{2}} \cos ^{2} w
$$

where

$$
\overline{\bar{D}}_{T(s)} \bar{\alpha}(s)=(\cos w) \overline{\bar{D}}_{T(s)} \alpha(s)+\frac{a}{-a^{2}+c^{2}}(\cos w) \overline{\bar{D}}_{T(s)} X(s)
$$

If

$$
-a^{2}+c^{2}>0
$$

that is

$$
\left\langle\overline{\bar{D}}_{T(s)} \bar{\alpha}(s), \overline{\bar{D}}_{T(s)} \bar{\alpha}(s)\right\rangle<0,
$$

then $\bar{\alpha}(s)$ is timelike curve and similarly, if

$$
-a^{2}+c^{2}<0
$$

that is

$$
\left\langle\overline{\bar{D}}_{T(s)} \bar{\alpha}(s), \overline{\bar{D}}_{T(s)} \bar{\alpha}(s)\right\rangle>0,
$$

then $\bar{\alpha}(s)$ is spacelike curve.

\section{Dispersion parameter}

Let the base curve of a $T S$-geodesic ruled surface $M$ be the striction curve in de-Sitter space $S_{1}^{3}$. Then, the distance from the striction point to the base curve is

$$
w=\arctan \left(\frac{a}{-a^{2}+c^{2}}\right)=0 .
$$

Hence, we have

$$
a=0
$$

and since

$$
\bar{D}_{T(s)} X(s)=a T(s)+c \xi(s)
$$

the vector field $\bar{D}_{T(s)} X(s)$ and normal of surface $\xi(s)$ are linearly independent. Therefore, there exists $\lambda \in \mathbb{R}$ for the equality

$$
\xi(s)=\lambda \bar{D}_{T(s)} X(s) .
$$

On the other hand, since

$$
\xi(s)=\varphi \times X \times T
$$

and

$$
\varphi=(\cos t) \alpha(s)+(\sin t) X(s),
$$

we have

$$
\xi(s)=(\cos t)[\alpha(s) \times X(s) \times T(s)]
$$


Therefore, we have

$$
\lambda \bar{D}_{T(s)} X(s)=(\cos t)[\alpha(s) \times X(s) \times T(s)] .
$$

If we take the scalar product with $\bar{D}_{T(s)} X(s)$ of both sides of the above equality, then we have

$$
\lambda=(\cos t) \frac{\operatorname{det}\left(\alpha(s), T(s), X(s), \bar{D}_{T(s)} X(s)\right)}{\left\langle\bar{D}_{T(s)} X(s), \bar{D}_{T(s)} X(s)\right\rangle},
$$

where $\lambda$ is called a dispersion parameter of $T S$-geodesic ruled surface in de-Sitter space $S_{1}^{3}$.

Example 6.1. The surface of example-1 above is an example of a developable ruled surface in de-Sitter space $S_{1}^{3}$. It is clear that for timelike geodesic ruled surface $M$ in de-Sitter space $S_{1}^{3}$ given by parametrization

$$
\varphi: I \times \mathbb{R} \rightarrow S_{1}^{3}, \varphi(s, t)=(\cos t) \alpha(s)+(\sin t) X(s)
$$

if

$$
\alpha(s)=(\sinh s, 0 \cosh s, 0)
$$

and

$$
X(s)=(-\cosh s, \sqrt{2}, \sinh s, 0)
$$

are chosen, then we can derive

$$
\operatorname{det}\left(T, X, \varphi, \overline{\bar{\Delta}}_{T} X\right)=0
$$

Therefore

$$
\lambda=(\cos t) \frac{\operatorname{det}\left(\alpha(s), T(s), X(s), \bar{\Delta}_{T(s)} X(s)\right)}{\left\langle\bar{\Delta}_{T(s)} X(s), \bar{\Delta}_{T(s)} X(s)\right\rangle}=0 .
$$

Theorem 6.2. The TS-geodesic ruled surface $M$ in de-Sitter space $S_{1}^{3}$ is developable if and only if the dispersion parameter of $M$ is zero.

Proof. From Corollary-1 and Corollary-2, we get

$$
c=-\operatorname{det}\left(T(s), X(s), \alpha(s), \overline{\bar{D}}_{T(s)} X(s)\right)=0 .
$$

It is clear from the definition of the dispersion parameter that

$$
\lambda=(\cos t) \frac{\operatorname{det}\left(\alpha(s), T(s), X(s), \bar{D}_{T(s)} X(s)\right)}{\left\langle\bar{D}_{T(s)} X(s), \bar{D}_{T(s)} X(s)\right\rangle}=0 .
$$

Definition 6.3. If there exists a curve that cuts vertically each main geodesic of the TS-geodesic ruled surface in de-Sitter space $S_{1}^{3}$, then this curve is called orthogonal trajectory of $T S$-geodesic ruled surface in de-Sitter space $S_{1}^{3}$.

Theorem 6.4. Let $M$ be a TS-geodesic ruled surface in de-Sitter space $S_{1}^{3}$. There is only one orthogonal trajectory which passes through every point of $M$.

Proof. Let $M$ be a $T S$-geodesic ruled surface given by the parametrization $\varphi: I \times J \rightarrow S_{1}^{3} \subset R_{1}^{4}$,

$$
\varphi(s, t)=(\cos t) \alpha(s)+(\sin t) Z(s) .
$$

Then, the orthogonal trajectory of $M$ is $\beta: \tilde{I} \subset I \rightarrow M$,

$$
\beta(s)=[\cos f(s)] \alpha(s)+[\sin f(s)] Z(s) .
$$

Since

$$
\left\langle\bar{D}_{T(s)} \beta(s), Z(s)\right\rangle=0,
$$


we get

$$
f(s)=-\int\left\langle\overline{\bar{D}}_{T(s)} \alpha(s), Z(s)\right\rangle d s+h
$$

where $\langle Z(s), Z(s)\rangle=1$. If we take

$$
F(s)=-\int\left\langle\overline{\bar{D}}_{T(s)} \alpha(s), Z(s)\right\rangle d s
$$

we get

$$
f(s)=F(s)+h .
$$

Since $h$ is chosen arbitrary, there are a lot of curves that satisfy the condition

$$
\left\langle\bar{D}_{T(s)} \beta(s), Z(s)\right\rangle=0 .
$$

Let us now find $s \in \mathbb{R}$ such that

$$
P_{0}=[\cos (F(s)+h)] \alpha(s)+[\sin (F(s)+h)] Z(s) .
$$

This leads to

$$
[\cos f(s)] \alpha(s)+[\sin f(s)] Z(s)=\left[\cos v_{0}\right] \alpha\left(s_{0}\right)+\left[\sin v_{0}\right] Z\left(s_{0}\right)
$$

So,

$$
\alpha\left(s_{0}\right)=\alpha(s), v_{0}=f(s)
$$

If we choose interval $I$ such that $\alpha$ is one to one, then we get

$$
s=s_{0}
$$

Thus,

$$
h=f\left(s_{0}\right)-F\left(s_{0}\right) \text {. }
$$

Consequently, there exists only one orthogonal trajectory passing through the point $P_{0}$. Therefore, $\tilde{I}$ must be equal to $I$.

Theorem 6.5. Let $M$ be undevelopable TS-geodesic ruled surface in de-Sitter space $S_{1}^{3}$. The shortest distance along the orthogonal trajectory between of any two main geodesics of $M$ is the distance measured along curve $\varphi_{t}: I \rightarrow M$ corresponding to

$$
t=\frac{1}{2} \arctan \left(\frac{2 a}{1-a^{2}+c^{2}}\right)
$$

Proof. Let us take two geodesics passing through points $\alpha\left(s_{1}\right)$ and $\alpha\left(s_{2}\right)$ where $s_{1}, s_{2} \in I$ and $s_{1}<s_{2}$. Also, let us denote distance obtained along orthogonal trajector $t=$ constant between these lines by $d(t)$. Then,

$$
d(t)=\int_{s_{1}}^{s_{2}}\|A\| d s=\sqrt{-(\cos t+a \sin t)^{2}+c^{2} \sin ^{2} t}\left(s_{2}-s_{1}\right),
$$

where

$$
A=(\cos t+a \sin t) T(s)+c(\sin t) \xi(s) .
$$

If $d^{\prime}(t)=0$, then $d(t)$ takes minimum value. Hence we get

$$
t=\frac{1}{2} \arctan \left(\frac{2 a}{1-a^{2}+c^{2}}\right) .
$$

Theorem 6.6. Let M be TS-geodesic ruled surface in de-Sitter space $S_{1}^{3}$. The geodesic of $M$ is both asymptotic and geodesic curves. 
Proof. Let $X$ be a tangent vector field of a geodesic of a $T S$-geodesic ruled surface $M$. Since every geodesic in ruled surface $M$, it is a geodesic $S_{1}^{3}$. Thus we get

$$
\bar{D}_{X} X=0
$$

From [9], we also get

$$
\bar{D}_{X} X=D_{X} X-\langle S(X), X\rangle \xi
$$

Thus

$$
D_{X} X=\langle S(X), X\rangle \xi
$$

Therefore

$$
D_{X} X \in \chi(M) \text { and }\langle S(X), X\rangle \xi \in \chi^{\perp}(M) .
$$

Since the metric on $M$ is nondegenerate, we get

$$
\chi\left(S_{1}^{3}\right)=\chi(M) \oplus \chi^{\perp}(M) \text { and } \chi(M) \cap \chi^{\perp}(M)=\{0\} .
$$

Thus

$$
D_{X} X=0 \text { and }\langle S(X), X\rangle=0 .
$$

The proof is completed.

Theorem 6.7. Let $M$ be $T S$-geodesic ruled surface in de-Sitter space $S_{1}^{3}$. Then

$$
K(p) \geq 0 \text { for all } p \in M
$$

where $K$ is the Gauss curvature function of $M$.

Proof. Let $X$ be the tangent vector field of the main geodesic at point $p \in M$ and take the orthonormal basis $\{X, Y\}$ of $\chi(M)$. Since $M$ is a timelike ruled surface, $X, Y$ are timelike and spacelike vector fields, respectively. The Weingarten operator $S$ of $M$ can be written

$$
\begin{aligned}
& S(X)=-\langle S(X), X\rangle X+\langle S(X), Y\rangle Y \\
& S(Y)=-\langle S(Y), X\rangle X+\langle S(Y), Y\rangle Y
\end{aligned}
$$

In this case, the matrix

$$
S=\left[\begin{array}{ll}
-\langle S(X), X\rangle & \langle S(X), Y\rangle \\
-\langle S(Y), X\rangle & \langle S(Y), Y\rangle
\end{array}\right]
$$

is corresponding to Weingarten operator $S$. On the other hand, the Weingarten operator $S$ is selfadjoint,

$$
\langle S(Y), X\rangle=\langle Y, S(X)\rangle
$$

Also, by Theorem 6.6, we conclude

$$
\langle S(X), X\rangle=0,\langle S(Y), Y\rangle=0 .
$$

Hence, from the definition of Gauss curvature, we get

$$
K=\operatorname{det} S=\langle S(X), Y\rangle^{2} .
$$

The proof is completed.

Theorem 6.8. Let $M$ be a TS-geodesic ruled surface in de-Sitter space $S_{1}^{3}$. Then

$$
\left\{\begin{array}{l}
\varphi \times T \times X=\xi \\
T \times X \times \xi=-\varphi \\
\xi \times \varphi \times T=-X \\
X \times \xi \times \varphi=-T
\end{array}\right.
$$

where $T$ is a unit tangent vector of base curve, $\varphi$ is the position vector of $M, X$ is unit tangent vector field of the main geodesic of $M$ and $\xi$ is unit normal vector field of $M$. 


\section{Acknowledgements}

The authors would like to express their sincere thanks to the editor and the anonymous reviewers for their helpful comments and suggestions.

\section{Funding}

There is no funding for this work.

\section{Availability of data and materials}

Not applicable.

\section{Competing interests}

The authors declare that they have no competing interests.

\section{Author's contributions}

All authors contributed equally to the writing of this paper. All authors read and approved the final manuscript.

\section{References}

[1] A. Turgut, H. Hacısalihoğlu, Timelike ruled surface in the Minkowski-3 space, Far East J. Math. Sci., 5(1)(1997), 83-90.

[2] A. Turgut, Spacelike and Timelike Ruled Surface on the Minkowski 3-Space, Ph. D. Thesis, Ankara University, 1995.

[3] A. Sabuncuoğlu, Generalized ruled surface, Associate Professorship Thesis, Ankara University, 1982.

[4] T. Mert, Spacelike ruled surfaces in hyperbolic 3-Space, Cumhuriyet Sci. J., 39(2)(2018), 314-324.

[5] J. Ratcliffe, Foundations of Hyperbolic Manifolds, New York, USA, 2006.

[6] B. O'Neill, Semi-Riemannian Geometry with applıcations to relativity, Academic Press, New York, 1983.

[7] M. Kasedou, Spacelike submanifolds in de-Sitter space, Demonstratio Mathematica, XLIII (2010), 401-418.

[8] T. Mert, B. Karlığa, Constant angle aurface in hyperbolic and de-Sitter 3-spaces, Ph. D. Thesis, Gazi University, 2014.

[9] C. Thas, A gauss map on hypersurfaces of submanifolds in Euclidean spaces, J.Korean Math. Soc., 16(1) (1979), 17-27.

[10] B. Karlığa, On the generalized stereographic projection, Beitr Algebra Geom., 37(2)(1996), 329-336. 